activated microglia populated the area in which neurons had disappeared.

Conclusions Acute development of NEC is associated with neuron loss, microglial activation, and increased IL-8 levels in the hippocampus of preterm pigs. Gut inflammatory disorders and increased intestinal permeability may affect the immature brain and contribute to long term neurological disorders.

\section{0-216 MELATONINE REDUCES BBB BREAKDOWN IN A RAT MODEL OF NEONATAL EXCITOTOXIC DAMAGE}

${ }^{1}$ A Zanin, ${ }^{2} \mathrm{R}$ Moretti, ${ }^{2} \mathrm{~J}$ Pansiot, ${ }^{2} \mathrm{D}$ Spiri, ${ }^{2} \mathrm{~L}$ Manganozzi, ${ }^{3} \mathrm{~K}$ Kratze, ${ }^{4} \mathrm{G}$ Favero, ${ }^{3} \mathrm{~A}$ Vasiljevic, ${ }^{2}$ VE Rinaldi, ${ }^{2}$ Pic, ${ }^{2} \mathrm{D}$ Massano, ${ }^{2} \mathrm{D}$ D'Agostino, ${ }^{2} \mathrm{MR}$ La Rocca, ${ }^{4} \mathrm{~L}$ Rodella, ${ }^{4} \mathrm{R}$ Rezzani, ${ }^{5} \mathrm{~J}$ Ek, ${ }^{3} \mathrm{~N}$ Strazielle, ${ }^{3} \mathrm{JF}$ Ghersi-Egea, ${ }^{2} \mathrm{P}$ Gressens, ${ }^{2} \mathrm{~L}$ Titomanlio. ${ }^{1}$ Neonatal Intensive Care Unit, Robert Debre Hospital, Paris, France; ${ }^{2}$ INSERM UMR-676, Robert Debre Hospital, Paris, France; ${ }^{3}$ Inserm U1028 CNRS UMR5292, Lyon Neurosciences Research Center, Lyon, France; ${ }^{4}$ Department of Clinical and Experimental Sciences, Section of Anatomy and Physiopathology, Brescia, Italy; ${ }^{5}$ Perinatal Center Dept Neuroscience and Physiology, Gothenburg University, Gothenburg, Sweden

\subsection{6/archdischild-2014-307384.286}

Objective The Blood-brain barrier (BBB) is a complex structure that protects the central nervous system (CNS) extracellular fluid from peripheral insults. Understanding the molecular basis and functioning of the BBB has a significant potential for future strategies to prevent and treat neurological disorders. The aim of our study was (1) to investigate BBB alterations in an excitotoxic model and (2) to test the protective properties of melatonin.

Methods The glutamate analogue ibotenate was injected intracerebrally in postnatal day 5 (P5) rat pups to mimic excitotoxic injury. Rats were sacrificed at $\mathrm{P} 5+2 \mathrm{~h}, \mathrm{P} 5+4 \mathrm{~h}, \mathrm{P} 5+18 \mathrm{~h}$. Lesion size and location of tight junction (TJ) proteins were determined by immunohistochemistry and BBB leakage after ibotenate injection by dextran staining. BBB proteins gene expression (TJs efflux transporters and detoxification enzymes) was determined on cortex and plexus. A group of pups was treated with melatonin $(5 \mathrm{mg} / \mathrm{kg}$, intraperitoneal).

Results Dextran extravasation was found $2 \mathrm{~h}$ after the insult, suggesting a rapid $\mathrm{BBB}$ breakdown that resolved at $+4 \mathrm{~h}$. A significant reduction in extravasation was observed in melatonintreated pups. Molecular Biology, immunohistochemistry and electron microscopy showed a dynamic BBB modification during the first $4 \mathrm{~h}$, partially reversed with melatonin. Lesion size evaluation confirmed melatonin white matter neuroprotection.



Abstract 0-216 Figure 1 A quantitative analysis of lesion size in cortical plate and white matter induced by ic injection of ibotenate (ibo) in the presence or in the absence of ip melatonin injection $5 \mathrm{mg} / \mathrm{kg}$

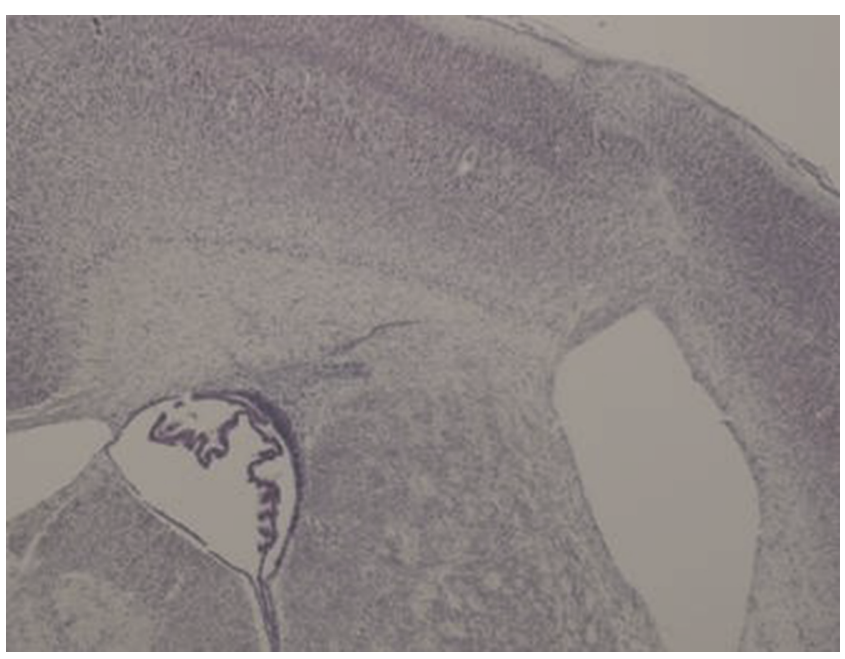

Abstract 0-216 Figure 2 B, C Cresyl-violet stained sections showing brain lesions induced by ibotenate ic injected on P5 rat pups and studied at the age of P10. (B: PBS ip injection, C:5 mg/kg melatonin ip injection)

Interpretation Our study, for the first time, evaluates the BBB at a very early time point, and it demonstrates that excitotoxicity causes early BBB disruption and that at this phase melatonin neuroprotects by preventing TJ proteins modifications, before acting as an anti-inflammatory and antioxidant molecule, and promoting axonal regrowth.

\section{0-217 ASSESSMENT OF MYOCARDIAL FUNCTION IN PRETERM INFANTS WITH CHRONIC LUNG DISEASE USING TISSUE DOPPLER IMAGING}

P Yajamanyam, RJS Negrine, SV Rasiah, AK Ewer. Neonatology, Birmingham Women's NHS Foundation Trust, Birmingham, UK

\subsection{6/archdischild-2014-307384.287}

Background Chronic lung disease (CLD): oxygen requirement at 36 weeks corrected gestational age (CGA) is a significant neonatal morbidity which can have adverse effects on cardiac function until pre-school age. ${ }^{1}$ Conventional echocardiographic techniques such as fractional shortening (FS) and left ventricular output (LVO) may not identify cardiac dysfunction in preterm infants. We have previously demonstrated that tissue Doppler imaging (TDI) is useful in assessment of myocardial function in these patients. ${ }^{2}$

Objectives To compare myocardial function in preterm infants born at.

Methods 50 preterm infants with CLD (25 receiving low flow nasal cannula oxygen and 25 receiving non-invasive positive airway pressure) and 22 without CLD (controls) had an echocardiogram at approximately 36 weeks CGA. Myocardial function was evaluated using FS, LVO and TDI. Ethical approval and written parental consent were obtained.

Results Median GA and birth weight of infants with CLD was lower than controls (27 wk (23-31) vs. 29 wk (23-31); $829 \mathrm{~g}$ (500-1790) vs.1030 g (570-1700)). There was no difference in persistence of PDA, tricuspid regurgitation, left ventricular FS and LVO between the groups. However, using TDI right ventricular peak systolic (S') and late diastolic velocities (A') $(p<0.001)$ were all significantly higher in CLD cases compared with controls.

Conclusion Cardiac dysfunction in this vulnerable group of patients can be better identified with TDI compared to FS and 
LVO. Using TDI may improve the identification of cardiac dysfunction and guide further management.

\section{REFERENCES}

1 Koroglu OA et al. Neonatology 2013;104(4):283-289

2 Negrine RJS et al. Arch Dis Child Fetal Neonatal Ed 2012;97 F304-F306

\section{$0-218$ THE EFFECT OF CAFFEINE ON DIAPHRAGMATIC ACTIVITY IN PRETERM INFANTS}

${ }^{1} \mathrm{JV}$ Kraaijenga, ${ }^{1} \mathrm{GJ}$ Hutten, ${ }^{2} \mathrm{LA}$ van Eykern, ${ }^{1} \mathrm{FH}$ de Jongh, ${ }^{1} \mathrm{AH}$ van Kaam. ${ }^{1}$ Neonatology, Emma Children's Hospital/Academic Medical Center, Amsterdam, Netherlands, ${ }^{2}$ Research and Development, Inbiolab BV, Groningen, Netherlands

\subsection{6/archdischild-2014-307384.288}

Background Preterm infants born with a GA $<32$ weeks are at high risk of developing central apnea of prematurity (AOP). Treatment with caffeine reduces central AOP by stimulating the breathing centre. Animal studies suggest that caffeine improves contractility of the diaphragm. We have determined the effect of caffeine on diaphragmatic activity in preterm infants.

Methods Spontaneously breathing preterm infants $<32$ weeks treated with an intravenous loading dose $(10 \mathrm{mg} / \mathrm{kg})$ of caffeine base for central AOP were eligible for the study. Diaphragmatic activity was continuously measured by transcutaneous electromyography (dEMG) starting 30-min before (baseline) until 1-hour after caffeine administration. Diaphragmatic inspiratory activity per breath, expressed as the relative amplitude change of dEMG (logEMGAR), area under the curve (AUC), respiratory rate (RR), as well as tidal volume $\left(\mathrm{V}_{\mathrm{t}}\right)$ measured by respiratory inductive plethysmography, were calculated at 4 fixed time points after caffeine administration (5,15,30 and 60-min) using the average of all breaths in a 30-sec recording and compared to baseline.

Results 30 preterm infants (mean GA $29.1 \pm 1.3$ wk; birth weight $1237 \pm 370 \mathrm{~g}$ ) were included. 5-min after caffeine administration, diaphragmatic activity significantly increased (median, IQR) compared to baseline; logEMGAR (0.13, 0.09$0.17)$, corresponding with an amplitude increase of 35\% (22$49 \%)$. AUC (19\%, 11-34\%) and $\mathrm{V}_{\mathrm{t}}(30 \%, 7-48)$ also increased significantly. Caffeine did not impact RR. The increased activity was observed at all subsequent time points.

Conclusions This is the first study showing that caffeine treatment, besides stimulating respiratory drive, results in a rapid (within 5-min) and sustained increase in diaphragmatic contractility in preterm infants.

\section{Late Breaking}

\section{0-219 DEVELOPING AND EVALUATING AN ON LINE PARENT INFORMATION AND SUPPORT APPLICATION TO FACILITATE HOME-BASED CARE BY PARENTS OF LONG-TERM CONDITIONS: A FEASIBILITY RCT}

${ }^{1}$ V Swallow, ${ }^{1}$ I Carolan, ${ }^{1} \mathrm{~A}$ Hall, ${ }^{2} \mathrm{~T}$ Smith, ${ }^{2} \mathrm{NJA}$ Webb, ${ }^{1} \mathrm{M}$ Campbell, ${ }^{3} \mathrm{~K}$ Knafl, ${ }^{3} \mathrm{~S}$ Santacroce, ${ }^{1} \mathrm{M}$ Harper-Jones. 'School of Nursing Midwifery and Social Work, University of Manchester, Manchester, UK; ${ }^{2}$ Nephrology, Royal Manchester Children's Hospital, Manchester, UK; ${ }^{3}$ School of Nursing, University of North Carolina at Chapel Hill, Chapel Hill, USA

\subsection{6/archdischild-2014-307384.289}

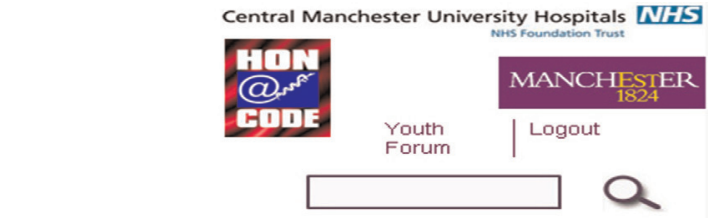

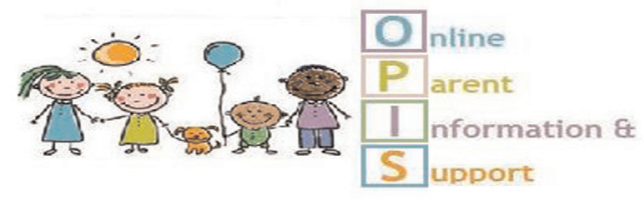

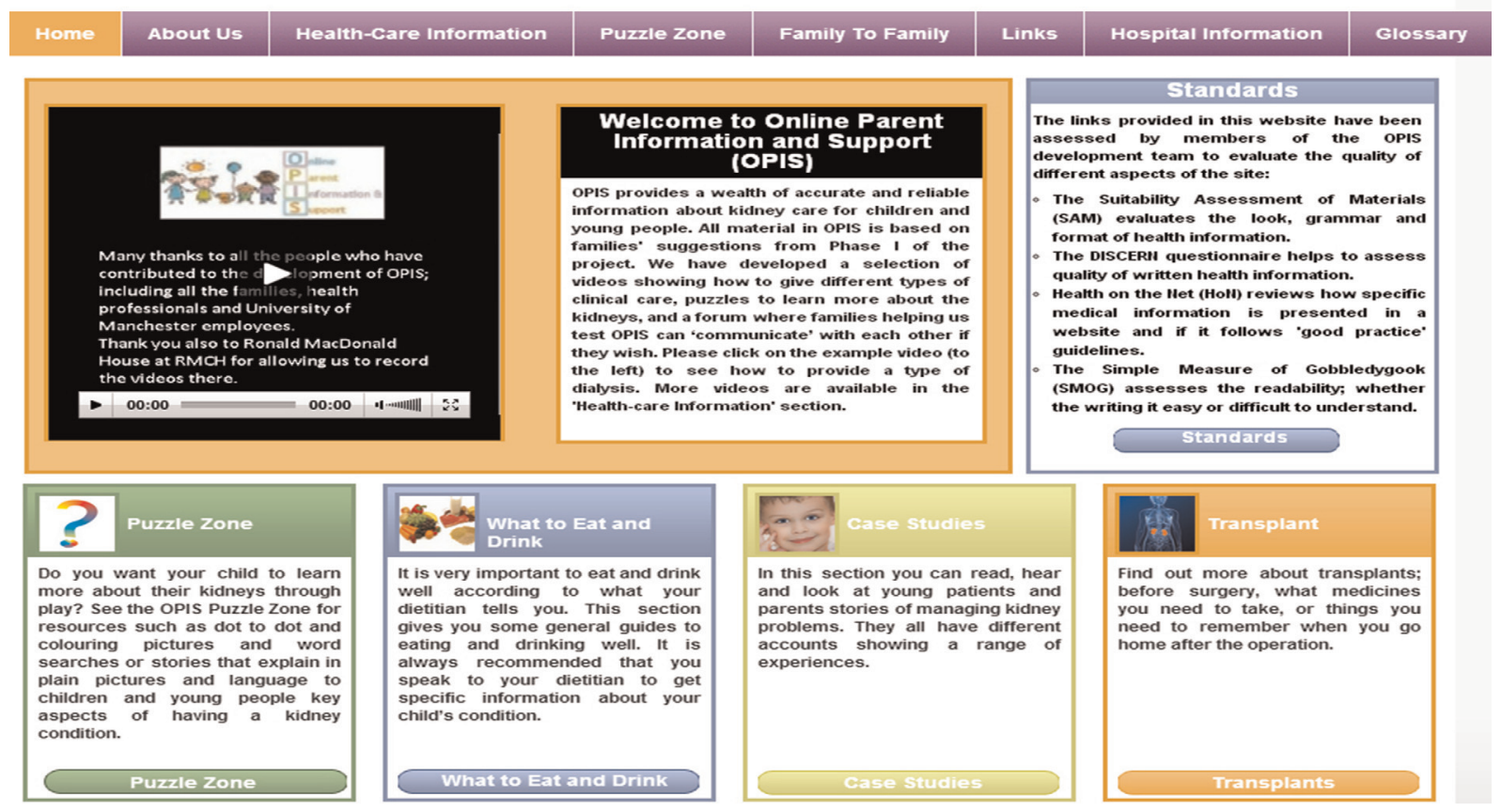

Abstract 0-219 Figure 1 